\title{
Cardiac Magnetic Resonance Imaging in Patients with Acute Ischemic Stroke and Elevated Troponin: A TRoponin ELevation in Acute Ischemic Stroke (TRELAS) Sub-Study
}

\author{
Karl Georg Haeusler ${ }^{a} \quad$ Christoph Jensen ${ }^{b} \quad$ Jan F. Scheitz ${ }^{c, d}$ \\ Thomas Krause $^{e}$ Christian Wollboldt ${ }^{c}$ Bernhard Witzenbichler ${ }^{f}$ \\ Heinrich J. Audebert ${ }^{c, d}$ Ulf Landmesser ${ }^{g-i}$ Jochen B. Fiebach ${ }^{d}$ \\ Christian H. Nolte ${ }^{c, d, h}$ Matthias Endres ${ }^{c, d, h-j}$ \\ Hans-Christian Mochmann ${ }^{9}$ \\ aDepartment of Neurology, Universitätsklinikum Würzburg, Würzburg, Germany; \\ ${ }^{b}$ Department of Cardiology, Marienhospital Gelsenkirchen, Gelsenkirchen, Germany; \\ 'Department of Neurology, Charité - Universitätsmedizin Berlin, Berlin, Germany; \\ ${ }^{d}$ Center for Stroke Research Berlin, Charité - Universitätsmedizin Berlin, Berlin, Germany; \\ e Department of Neurology, Jüdisches Krankenhaus Berlin, Berlin, Germany; ${ }^{\mathrm{f}}$ Department of \\ Cardiology and Pneumology, Helios Amper-Klinikum Dachau, Dachau, Germany; \\ gDepartment of Cardiology, Charité - Universitätsmedizin Berlin, Campus Benjamin Franklin, \\ Berlin, Germany; ${ }^{\text {h} B e r l i n ~ I n s t i t u t e ~ o f ~ H e a l t h ~(B I H), ~ B e r l i n, ~ G e r m a n y ; ~}{ }^{i}$ German Center for \\ Cardiovascular Diseases (DZHK), Partner Site Berlin, Berlin, Germany; ${ }^{j}$ German Center for \\ Neurodegenerative Diseases (DZNE), Partner Site Berlin, Berlin, Germany
}

\section{Keywords}

Magnetic resonance imaging $\cdot$ Stroke $\cdot$ Acute coronary syndrome $\cdot$ Coronary angiography

\begin{abstract}
Background: Elevated high-sensitive cardiac troponin (hs-cTn) can be found in more than $50 \%$ of the patients with acute ischemic stroke. The observational TRoponin ELevation in Acute ischemic Stroke (TRELAS) study revealed that about $25 \%$ of all stroke patients with elevated troponin had a coronary angiography-detected culprit lesion affording immediate intervention, and about $50 \%$ of all patients did not have any obstructive coronary artery disease. Given the risk of procedure-related complications, the identification of stroke patients in urgent need of invasive coronary angiography is desirable. Methods: TRELAS patients were prospectively enrolled into this sub-study. In addition to conventional coronary angiography,
\end{abstract}

Matthias Endres and Hans-Christian Mochmann contributed equally to this work. Clinical trial registration: NCT01263964. 
a cardiac magnetic resonance imaging (MRI) at 3T was performed during the in-hospital stay after acute ischemic stroke to compare the diagnostic value of both imaging modalities. $\boldsymbol{R e}$ sults: Nine stroke patients (median age 73 years [range 58-87]; four females; median NIH Stroke Severity score on admission 4 [range 0-6] with elevated hs-cTnT [median $74 \mathrm{ng} / \mathrm{L}$, interquartile range 41-247] on admission) completed cardiac MRI and underwent coronary angiography. The absence of MRI-detected wall motion abnormalities and/or late gadolinium enhancement in 5 stroke patients corresponded with the exclusion of culprit lesions or significant coronary artery disease by coronary angiography. Four patients had abnormal MRI findings, whereof 2 showed evidence of myocardial infarction and in whom coronary angiography demonstrated a $>70 \%$ stenosis of a coronary artery. Conclusions: The TRELAS substudy indicates that noninvasive cardiac MRI may provide helpful information to identify stroke patients with or without acute coronary syndrome. Our findings might help to select stroke patients in urgent need of coronary angiography.

(C) 2019 The Author(s)

Published by S. Karger AG, Basel

\section{Introduction}

In more than $50 \%$ of all patients with acute ischemic stroke, high-sensitive cardiac troponin (hs-cTn) is above the cutoff to rule out myocardial infarction [1]. The recently published prospective observational TRoponin ELevation in Acute ischemic Stroke (TRELAS) study revealed that patients with acute ischemic stroke and elevated hs-cTnT were significantly less likely to have coronary culprit lesions than age- and gender-matched patients with non-ST-elevation acute coronary syndrome [2]. Overall, about $25 \%$ of all TRELAS patients had a coronary culprit lesion, whereas about $50 \%$ did not have any obstructive coronary artery disease (CAD). While conventional coronary angiography is the gold standard to detect $\mathrm{CAD}$, the required periprocedural application of heparin - increasing the risk of hemorrhagic transformation - exposure to radiation, procedure-related complications as well as the needed dual antiplatelet therapy after coronary intervention limit the feasibility in the acute phase of ischemic stroke. While noninvasive cardiac computed tomography could add information about underlying CAD [3], the disadvantage of exposure to radiation is also present. Subsequently, assessment of an underlying CAD is often incomplete in patients with acute ischemic stroke in clinical practice. Cardiac magnetic resonance imaging (MRI) is now considered the gold standard to assess cardiac tumors, myocarditis, cardiomyopathies, and subclinical coronary heart disease [4]. Feasibility and safety of noninvasive cardiac MRI in patients with acute ischemic stroke has already been demonstrated. However, routine use of cardiac MRI is limited due to the necessity to follow breath-hold instructions and by the restricted availability of cardiac MRI [5]. This study aimed at comparing the diagnostic value of cardiac MRI compared to coronary angiography in patients with acute ischemic stroke and elevated hs-cTn.

\section{Materials and Methods}

The design of the investigator-initiated, prospective observational TRELAS study was published previously $[2,6]$. The review board of the Charite approved the TRELAS study protocol and the prospective MRI sub-study. All subsequent TRELAS patients at the Charité were asked to join the sub-study. After providing written informed consent, 24 patients admitted within $72 \mathrm{~h}$ after stroke onset and in-hospital hs-cTnT $>50 \mathrm{ng} / \mathrm{L}$ (Roche, Mannheim, Germany) underwent diagnostic coronary angiography. Patients with creatinine $\geq 1.2 \mathrm{mg} / \mathrm{dL}$, 

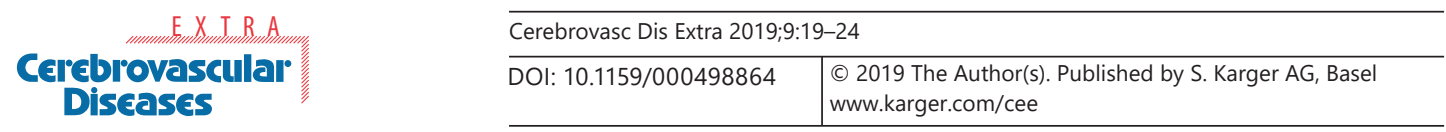

Haeusler et al.: Cardiac MRI in Stroke Patients with Elevated cTn

Table 1. Baseline characteristics, stroke localization, troponin levels (hs-cTnT), and ECG findings in 9 stroke patients of the TRELAS sub-study

\begin{tabular}{|c|c|c|c|c|c|c|c|c|}
\hline Sex & $\begin{array}{l}\text { Age, } \\
\text { years }\end{array}$ & $\begin{array}{l}\text { Stroke } \\
\text { localization }\end{array}$ & $\begin{array}{l}\text { NIHSS } \\
\text { admission }\end{array}$ & $\begin{array}{l}\text { Cardiovascular } \\
\text { risk factors }\end{array}$ & $\begin{array}{l}\text { Known } \\
\text { CAD }\end{array}$ & $\begin{array}{l}\text { hs-cTnT } \\
\text { admission }\end{array}$ & $\begin{array}{l}\text { hs-cTnT } \\
\text { follow-up }\end{array}$ & $\begin{array}{l}\text { Pathological ECG } \\
\text { findings on } \\
\text { admission }\end{array}$ \\
\hline M & 71 & multiple arteries & 0 & AHT, HC, smoking & no & $66 \mathrm{ng} / \mathrm{L}$ & $60 \mathrm{ng} / \mathrm{L}$ & none \\
\hline $\mathrm{F}$ & 82 & multiple arteries & 5 & $\mathrm{AF}, \mathrm{AHT}, \mathrm{HC}$ & no & $13 \mathrm{ng} / \mathrm{L}$ & $535 \mathrm{ng} / \mathrm{L}$ & signs of ischemia \\
\hline $\mathrm{F}$ & 71 & PCA left & 2 & $\mathrm{AF}, \mathrm{AHT}, \mathrm{HC}$ & no & $510 \mathrm{ng} / \mathrm{L}$ & $520 \mathrm{ng} / \mathrm{L}$ & $\mathrm{AF}$ \\
\hline M & 87 & MCA/PCA right & 4 & AF, AHT, HC & yes & $20 \mathrm{ng} / \mathrm{L}$ & $76 \mathrm{ng} / \mathrm{L}$ & $\mathrm{AF}$, signs of ischemia \\
\hline M & 73 & MCA/ACA left & 6 & AHT & no & $74 \mathrm{ng} / \mathrm{L}$ & 69 ng/L & none \\
\hline $\mathrm{F}$ & 75 & BA & 3 & AHT, HC & no & $95 \mathrm{ng} / \mathrm{L}$ & $128 \mathrm{ng} / \mathrm{L}$ & none \\
\hline M & 58 & multiple arteries & 4 & AF, AHT, HCM & no & $144 \mathrm{ng} / \mathrm{L}$ & $146 \mathrm{ng} / \mathrm{L}$ & $\begin{array}{l}\text { signs of ischemia, LV } \\
\text { hypertrophy }\end{array}$ \\
\hline M & 69 & MCA left & 4 & smoking & yes & $61 \mathrm{ng} / \mathrm{L}$ & $34 \mathrm{ng} / \mathrm{L}$ & LSB \\
\hline $\mathrm{F}$ & 79 & multiple arteries & 1 & $\begin{array}{l}\text { AF, AHT, HC, } \\
\text { diabetes }\end{array}$ & no & $347 \mathrm{ng} / \mathrm{L}$ & $518 \mathrm{ng} / \mathrm{L}$ & none \\
\hline
\end{tabular}

AHT, arterial hypertension; HC, hypercholesterolemia; AF, atrial fibrillation; HCM, hypertrophic cardiomyopathy; LV, left ventricular; LSB, least significant bit; PCA, posterior cerebral artery; MCA, middle cerebral artery; ACA, anterior cerebral artery; BA, basilar artery.

modified Rankin scale $\geq 4$ before admission, or ST-elevation at baseline echocardiography (ECG) were not enrolled. Nine stroke patients provided informed consent to undergo cardiac MRI at 3T (Magnetom Tim Trio; Siemens AG, Erlangen, Germany) as described previously [5]. ECG-gated images were acquired during breath hold using a phased array receiver coil (Body Matrix-coil\#TATS; Siemens AG). Cine images of three long-axis as well as 14-18 short-axis views using an ECG-gated gradient-echo sequence were acquired. Approximately $10 \mathrm{~min}$ after intravenous administration of $0.15 \mathrm{mmol} / \mathrm{kg}$ bodyweight Gadobutrol (Gadovist ${ }^{\circledR}$; Bayer HealthCare, Leverkusen, Germany) at a concentration of $1 \mathrm{mmol} / \mathrm{mL}$, an inversion recovery gradient-echo sequence was acquired in corresponding long-axis and short-axis slices adjusting the inversion time to null normal myocardium. Blinded cardiac MRI reading was done by a board-certified cardiologist (C.J.) specialized in cardiac MRI. Data were summarized with absolute and relative frequencies of qualitative characteristics or medians and interquartile range (IQR) for quantitative variables.

\section{Results}

The median age of the 9 stroke patients undergoing cardiac MRI was 73 years (range 58-87), four were female. The median NIH Stroke Severity (NIHSS) score on admission was 4 (range 0-6), 3 patients had a history of CAD (Table 1). Additional information can be found in Table 2, also including data of the 15 TRELAS patients who either rejected or were unable to undergo cardiac MRI. Besides a higher median creatine kinase on admission in patients undergoing additional MRI, univariate analysis revealed no differences between both patient groups.

All 9 stroke patients completed the cardiac MRI as well as coronary angiography during the in-hospital stay. The median delay between hospital admission and cardiac MRI or coronary angiography was $83 \mathrm{~h}$ (IQR 68-106) or $71 \mathrm{~h}$ (IQR 45-89), respectively. In 5 stroke patients, combined analysis of wall motion and late gadolinium enhancement showed no substantial findings (Table 3 ). Correspondingly, no significant $(>70 \%)$ coronary artery stenosis was detected by coronary angiography. Four stroke patients had abnormal MRI 
Haeusler et al.: Cardiac MRI in Stroke Patients with Elevated cTn

Table 2. Baseline characteristics of TRELAS patients with or without cardiac MRI (adapted from [2])

\begin{tabular}{|c|c|c|c|}
\hline & $\begin{array}{l}\text { Cardiac MRI } \\
(n=9)\end{array}$ & $\begin{array}{l}\text { No cardiac MRI } \\
(n=15)\end{array}$ & $p$ \\
\hline Median age (IQR), years & $73(70-81)$ & 77 (64-82) & 0.770 \\
\hline Male sex & $55.6(5)$ & $60.0(9)$ & 1.0 \\
\hline Median NIHSS score (IQR) & $3(2-4)$ & $3(3-4)$ & 0.411 \\
\hline Median GRACE score (IQR) & $118(98-145)$ & $113(83-148)$ & 0.770 \\
\hline \multicolumn{4}{|l|}{ Cardiovascular risk factors } \\
\hline Diabetes mellitus & $22.2(2)$ & $26.7(4)$ & 1.0 \\
\hline Hypercholesterolemia & $77.8(7)$ & $46.7(7)$ & 0.210 \\
\hline Hypertension & $100(9)$ & $86.7(13)$ & 0.511 \\
\hline Previous stroke & $33.3(3)$ & $13.3(2)$ & 0.326 \\
\hline Current smoking & $22.2(2)$ & $13.2(2)$ & 0.615 \\
\hline Atrial fibrillation & $55.6(5)$ & $46.7(7)$ & 1.0 \\
\hline Chronic heart failure & $22.2(2)$ & $26.7(4)$ & 1.0 \\
\hline History of CAD & $22.2(2)$ & $20.0(3)$ & 1.0 \\
\hline \multicolumn{4}{|l|}{ Laboratory measures at baseline } \\
\hline Median hs-cTn levels (IQR), ng/L & $74(41-247)$ & $85(44-167)$ & 1.0 \\
\hline Median creatinine kinase (IQR), mg/dL & $166(117-1540)$ & $86(61-147)$ & 0.025 \\
\hline Median creatinine (IQR), mg/dL & $1.03(0.79-1.12)$ & $0.97(0.88-1.12)$ & 0.907 \\
\hline Median GFR (IQR), mL/min/1.73 m² & $72(63-76)$ & $71(59-85)$ & 0.907 \\
\hline Killip class & & & 0.172 \\
\hline 1 & $88.9(8)$ & $80.0(12)$ & \\
\hline 2 & $0(0)$ & $20.0(3)$ & \\
\hline 3 & $11.1(1)$ & $0(0)$ & \\
\hline \multicolumn{4}{|l|}{ Medication before admission } \\
\hline Prior antiplatelet use & $44.4(4)$ & $53.3(8)$ & 1.0 \\
\hline Prior oral anticoagulation & $11.1(1)$ & $6.7(1)$ & 1.0 \\
\hline Prior statin use & $44.4(4)$ & $20.0(3)$ & 0.356 \\
\hline Prior use of beta-blockers & $33.3(3)$ & $38.5(5)$ & 1.0 \\
\hline
\end{tabular}

Values are presented as \% $(n)$, unless otherwise indicated. GRACE, Global Registry of Acute Coronary Events; GFR, glomerular filtration rate estimated according to the CKD-EPI formula.

findings, whereof 2 patients showed evidence of myocardial infarction. In both patients, coronary angiography demonstrated pathological findings, including a $>70 \%$ stenosis of a corresponding coronary artery requiring stenting. Apical ballooning (stress cardiomyopathy) and a reduced cardiac ejection fraction was found in a single stroke patient by both imaging modalities. One patient showed nonischemic late gadolinium enhancement pathognomonic for hypertrophic cardiomyopathy (Table 3).

\section{Discussion}

This is the first prospective evaluation comparing the diagnostic value of 3-T cardiac MRI to coronary angiography in acute ischemic stroke patients with elevated hs-cTn. The exclusion of significant CAD by coronary angiography corresponded well with the absence of pathological MRI findings. Therefore, the assumption that cardiac MRI may help identify patients with or without need of invasive evaluation for CAD in the acute phase of ischemic stroke should be validated in a larger prospective study.

By providing information on myocardial infarction, cardiac MRI also appears to have a complementary diagnostic value to past medical history, laboratory results, echocardiog- 

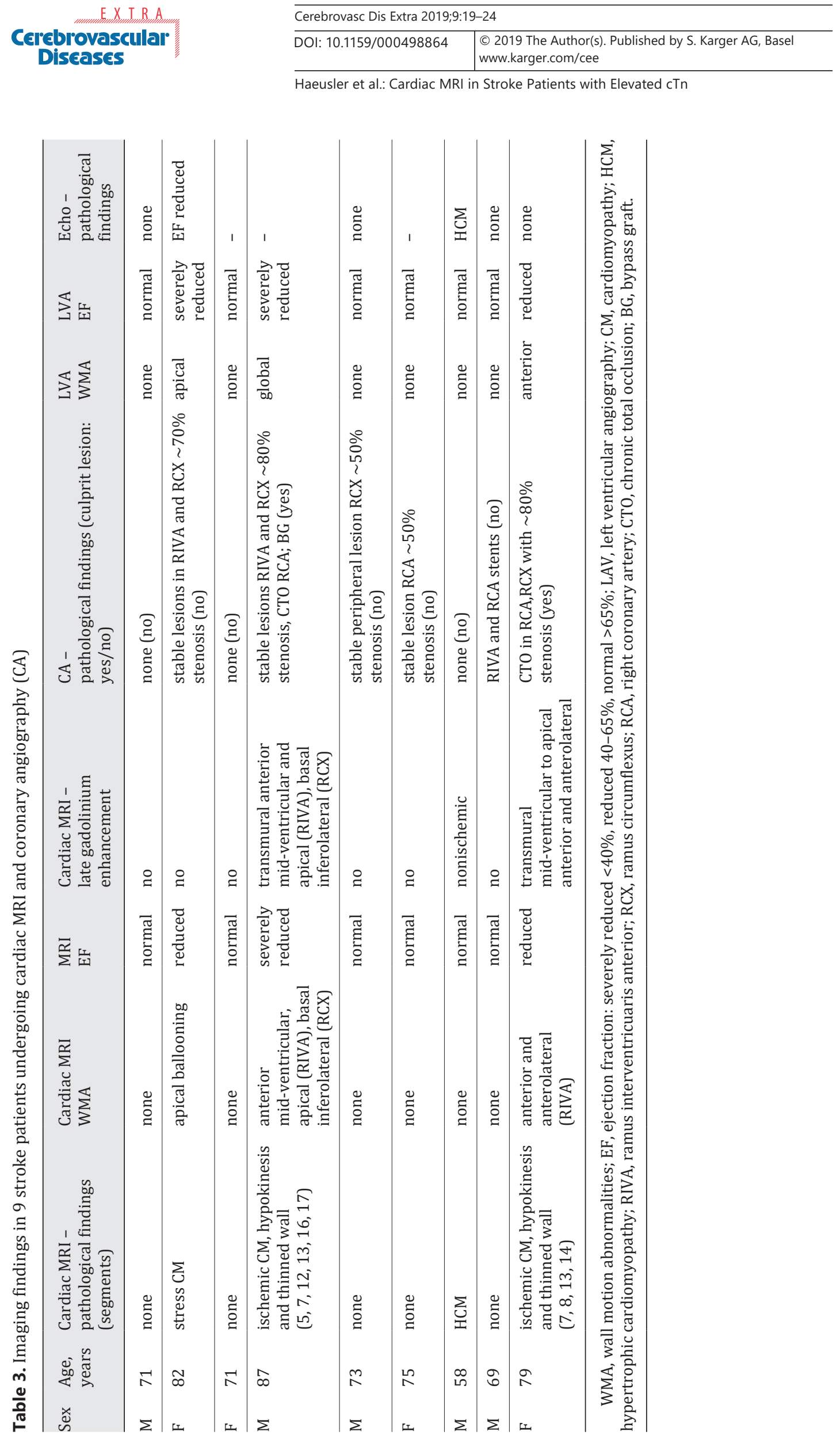
Haeusler et al.: Cardiac MRI in Stroke Patients with Elevated cTn

raphy, and ECG parameters in the assessment of acute coronary syndrome in stroke patients. However, cardiac MRI requires active patient cooperation, which cannot always be achieved in the acute phase of stroke [5] (Table 2).

\section{Acknowledgement}

We thank Julia Herde for critically reviewing the manuscript.

\section{Statement of Ethics}

The Ethics Committee of the Charité - Universitätsmedizin Berlin approved the TRELAS study protocol and the prospective MRI sub-study.

\section{Disclosure Statement}

K.G.H. reports honoraria from Bayer Healthcare, Sanofi, Pfizer, Boehringer Ingelheim, Bristol-Myers Squibb, Daiichi Sankyo, Medtronic, Biotronik, W. L. Gore \& Associates, and Edwards Lifesciences. C.J. reports lecture fees by Bayer Healthcare, Abbott Germany and Biotronik Germany, and research support by Novartis. J.F.S. reports lecture fees by W.L. Gore \& Associates. J.B.F. has received honoraria from Perceptive, BioClinica, Boehringer Ingelheim, Cerevast, Brainomix, and Lundbeck. H.J.A. has received a grant from Pfizer, honoraria from Boehringer Ingelheim, Bayer Healthcare, Sanofi, Daiichi-Sankyo, Pfizer, Bristol-Myers Squibb, Novo Nordisk, and EVER Neuropharma. C.H.N. reports honoraria from Boehringer Ingelheim, Bristol-Myers Squibb, Pfizer, Sanofi, and W.L. Gore \& Associates. M.E. reports grant support and/or fees paid to the Charité from Bayer, Boehringer Ingelheim, BMS/Pfizer, Daiichi Sankyo, Amgen, Sanofi, Covidien GSK, Ever, and Novartis, all outside the submitted work. H.-C.M. reports honoraria from Bayer Healthcare, Sanofi, Pfizer, and Daiichi Sankyo.

\section{Funding Sources}

The work was supported by funding from the Federal Ministry of Education and Research via the grant Center for Stroke Research Berlin (01 EO 0801).

\section{References}

1 Scheitz JF, Mochmann HC, Erdur H, Tütüncü S, Haeusler KG, Grittner U, et al. Prognostic relevance of cardiac troponin T levels and their dynamic changes measured with a high-sensitivity assay in acute ischaemic stroke: analyses from the TRELAS cohort. Int J Cardiol. 2014 Dec;177(3):886-93.

2 Mochmann HC, Scheitz JF, Petzold GC, Haeusler KG, Audebert HJ, Laufs U, et al.; TRELAS Study Group. Coronary Angiographic Findings in Acute Ischemic Stroke Patients With Elevated Cardiac Troponin: The Troponin Elevation in Acute Ischemic Stroke (TRELAS) Study. Circulation. 2016 Mar;133(13):1264-71.

3 Hur J, Choi BW. Cardiac CT Imaging for Ischemic Stroke: Current and Evolving Clinical Applications. Radiology. 2017 Apr;283(1):14-28.

4 Van Assche LM, Kim HW, Kim RJ. Cardiac MR for the assessment of myocardial viability. Methodist DeBakey Cardiovasc J. 2013 Jul-Sep;9(3):163-8.

5 Haeusler KG, Wollboldt C, Bentheim LZ, Herm J, Jäger S, Kunze C, et al. Feasibility and diagnostic value of cardiovascular magnetic resonance imaging after acute ischemic stroke of undetermined origin. Stroke. 2017 May;48(5):1241-7.

6 Scheitz JF, Mochmann HC, Nolte CH, Haeusler KG, Audebert HJ, Heuschmann PU, et al. Troponin elevation in acute ischemic stroke (TRELAS)—protocol of a prospective observational trial. BMC Neurol. 2011 Aug;11(1): 98. 